\title{
Business cases for assessing the value of active operation in distribution planning
}

DOI:

10.1049/cp.2012.2063

Link to publication record in Manchester Research Explorer

\section{Citation for published version (APA):}

Celli, G., Mocci, S., Ochoa, L. F., Pilo, F., \& Soma, G. G. (2012). Business cases for assessing the value of active operation in distribution planning. In IET Conference Publications/IET Conf Publ (Vol. 2012) https://doi.org/10.1049/cp.2012.2063

\section{Published in:}

IET Conference Publications|IET Conf Publ

\section{Citing this paper}

Please note that where the full-text provided on Manchester Research Explorer is the Author Accepted Manuscript or Proof version this may differ from the final Published version. If citing, it is advised that you check and use the publisher's definitive version.

\section{General rights}

Copyright and moral rights for the publications made accessible in the Research Explorer are retained by the authors and/or other copyright owners and it is a condition of accessing publications that users recognise and abide by the legal requirements associated with these rights.

\section{Takedown policy}

If you believe that this document breaches copyright please refer to the University of Manchester's Takedown Procedures [http://man.ac.uk/04Y6Bo] or contact uml.scholarlycommunications@manchester.ac.uk providing relevant details, so we can investigate your claim.

\section{OPEN ACCESS}




\title{
Business Cases for Assessing the Value of Active Operation in Distribution Planning
}

\author{
Gianni Celli, Susanna Mocci, Luis F. Ochoa, Fabrizio Pilo, Gian Giuseppe Soma
}

\begin{abstract}
Modern planning tools for active distribution networks should integrate network operation practices in the set of feasible planning alternatives, adopting probabilistic calculations (to capture the uncertain behavior of demand and generation) as well as multi-objective approaches. However, despite the recognized importance of active network solutions, most utilities do not consider such concepts as viable alternatives in their planning processes. Thus, utilities largely follow traditional 'fit and forget' planning approaches, i.e., only network reinforcements to cope with the worst case scenario. One of the reasons of this stagnation is the lack of ad hoc business cases that prove the benefits of active networks. Here, some business cases on real distribution networks have been prepared by using a novel planning tool to deal with all the uncertainties that characterize the future scenarios of distribution systems.
\end{abstract}

Index Terms-Active Distribution Networks, Distributed Energy Storage, Distributed Generation, Planning.

\section{INTRODUCTION}

$\mathrm{T}$ HE next decade will be characterized by electricity increasingly produced by or closer to consumers/load centers that are likely to feature higher levels of demand but also higher levels of responsiveness. Indeed, significant volumes of small-to-medium scale renewable generation capacity are expected to connect to the distribution networks (known as distributed generation, DG), resulting in bidirectional power flows. Additionally, the electrification of transport, particularly in the form of domestic-scale plug-in electric vehicles, and thermal needs (where applicable), could also become a reality. The growing need of greater observability, particularly for the end consumer, is also leading to the adoption of an advanced metering infrastructure that could in the future pave the way for responsive loads (e.g., appliances) from single or aggregated customers to be used to help managing both the distribution and transmission networks.

These and other potential changes in generation, load and the electricity infrastructure itself (such as the use of electricity storage) are part of the vision behind the need of a more intelligent power system (Smart Grid). The realization of this vision, however, places a number of technical challenges that if tackled in the traditional way might result in costly, underutilized assets or the inability to meet the

G. Celli, S. Mocci, F. Pilo and G. G. Soma are with the Department of Electrical and Electronic Engineering, University of Cagliari, Cagliari, 09123 ITALY (e-mail: celli@diee.unica.it, susanna.mocci@diee.unica.it, pilo@diee.unica.it,ggsoma@diee.unica.it).

L. F. Ochoa is with The University of Manchester, Manchester M13 9PL, UK (email: luis_ochoa@ieee.org) (carbon emission and/or renewable) targets.

In order to facilitate the integration of these low-carbon technologies, the use of a more active approach of managing distribution networks (including both network elements and participants) has been proposed in the last decade by academia and industry. Several works have been presented on the potential benefits of active distribution networks (ADNs) but mainly focusing on the operational challenges rather than planning aspects. The CIGRE Working Group C6.11 highlighted the fact that ADNs affect all planning activities as, for instance, DG affects load forecast and ADN solutions affect the design of the system. Consequently, Distribution Networks Operators (DNOs) need to move from the the passive planning paradigm towards one where integration -not simply connection- of distributed energy resources (DER) is taken account of at a reasonable cost [1]. The availability of communication, monitoring and control systems at a distribution level will make planning objectives to focus on the maximum exploitation of existing assets and infrastructures as it will be possible to operate them much closer to their physical limits than in the past. Future distribution network planning will in many cases result in IES toaditional network investments since operation issues can be fixed with the ADN solutions such as generator dispatch, demand side integration, control of transformer taps, reactive power management, and system reconfiguration. Therefore, ADN solutions will have a significant role in the optimal development of the distribution network and they have to be implemented in modern planning tools [2].

A recent survey carried out by CIGRE WG C6.19 to determine the state-of-the-art and identify which developments are needed for the planning of ADNs showed that $90 \%$ of the respondent utilities follow traditional steps of the typical planning process [3]. Although there are significant differences in how these activities are done, it is clear that, while of interest to many utilities, active distribution networks and demand side integration concepts fail to be considered by utilities as viable alternatives in the planning process. The reasons for this situation are the lack of planning tools able to deal correctly with future scenarios and the lack of ad hoc business cases that prove the corresponding benefits.

In this work, by using a novel planning tool for ADNs developed in [4], some business cases have been arranged based on a real distribution network in order to estimate the value of incorporating ADN solutions in distribution planning. 


\section{Accepted Paper}

\section{TRAditional PlanNing}

Distribution networks are, in general, sized to cope with the worst-case scenario (mainly in terms of loads and voltage drops, and certain security constraints) of a given load forecast and in a way that minimum or no operation is required. This approach, known as 'fit and forget', is carried out in a deterministic way, i.e., without considering uncertainties. Fig. 1 presents a generic flow chart that resembles this common practice. Once a planning study is defined, different alternatives might be considered. These are then technically assessed taking account of the (load) conditions for the corresponding planning horizon. If a planning alternative is not technically feasible (particular for the design of feeders), network reinforcements are applied. Otherwise, the next step is to evaluate the corresponding cost. The most cost-effective solution is finally the planning alternative likely to be adopted.

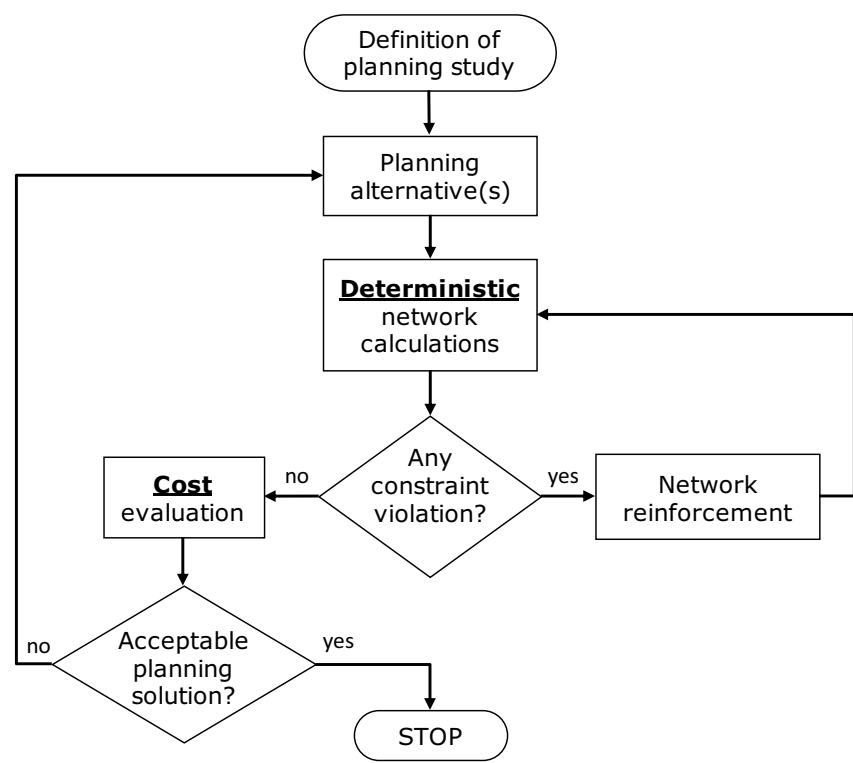

Fig. 1. General Planning Framework for Passive Networks.

When it comes to connecting distributed generation, the fit-and-forget approach is also applied. Although other relevant technical aspects such as congestion, voltage rise, reverse power flows, etc. are considered [5], it is mainly based on maximum generation-minimum demand scenarios that, for renewable sources, do not occur frequently. While this passive way of planning and operating distribution networks has proven cost-effective in the last decades, it might in the future become a barrier for increasing penetrations of DG and non-conventional loads.

Regulated or non-regulated DNOs will commonly provide firm capacity access to medium-scale DG plants (i.e., ability to produce up to the registered capacity at any time with a defined range of power factor capability), as long as there is minor or no impact on the network (otherwise reinforcements will need to be paid for according to national rules). This means that with each subsequent connection, the hosting capacity of the network will be reduced, reaching its fit-and-forget limit soon. Some DNOs, in order to facilitate further penetrations, have also adopted non-firm connections where generators are tripped automatically after a network constraint is breached. This approach can also reach its limits relatively soon either due to technical constraints or economic factors (for the DG developers).

In addition, the uncertainties in planning consents and financial support surrounding medium-scale DG investments pose DNOs with major challenges as to what, where and when to reinforce the system, in order to facilitate the transition to a low-carbon economy without the risk of stranded assets. This lack of certainty and planning coordination translates into DNOs often connecting DG plants in the aforementioned fit-and-forget, case-by-case manner. Thus, any sophisticated solution -albeit potentially more cost-effective for society in the long term- is left behind.

To overcome most of these inadequacies ADN solutions can be incorporated in the planning procedure in a way that uncertainties are also accounted for.

\section{Active Distribution Network Planning}

New distribution planning procedures have to be developed that are able to cope with all the uncertainties introduced by particularly DG. Moreover, these new tools must be scalable to deal with large distribution networks and should also include ad hoc business cases. It is with these characteristics DNOs will be prepared to adopt such a new planning tool.

The key feature of a modern planning tool for ADNs is the integration of future network operation practices in the set of feasible planning alternatives in order to identify the best technical and economic balance between an innovative solution (that tends to maximize asset utilization) and traditional network expansion. To this end, the representation of loads and generators cannot be based on a snapshot of the operating conditions (e.g., max generation/min demand, $\min$ generation/max demand) as commonly assumed by current planning methods and tools. There is the need of adopting time-series (or time dependent) models in order to capture the operational aspects that can affect the planning stage. Moreover, calculations should be based on probabilistic approaches to capture the uncertain behavior of the demand and the generation. In addition, the assessment/comparison of alternatives can also be based on one or more objectives.

Fig. 2 shows a general planning framework for ADN planning [1] even though not yet fully exploited by DNOs [3]. The following are the corresponding steps:

a) Input data (technical, financial, economic, etc.);

b) Pre-processing of ADN customer data (loads and generators) to capture time-dependent features;

c) Generation of a set of possible alternatives;

d) Making each alternative technically sound. If operation issues are expected in the planning period, try to solve them with operation. If this is not possible, resort to traditional network solutions;

e) Assessment of alternative, possibly with a multiobjective approach; and,

f) Selection of the best alternative or the set of best options. 


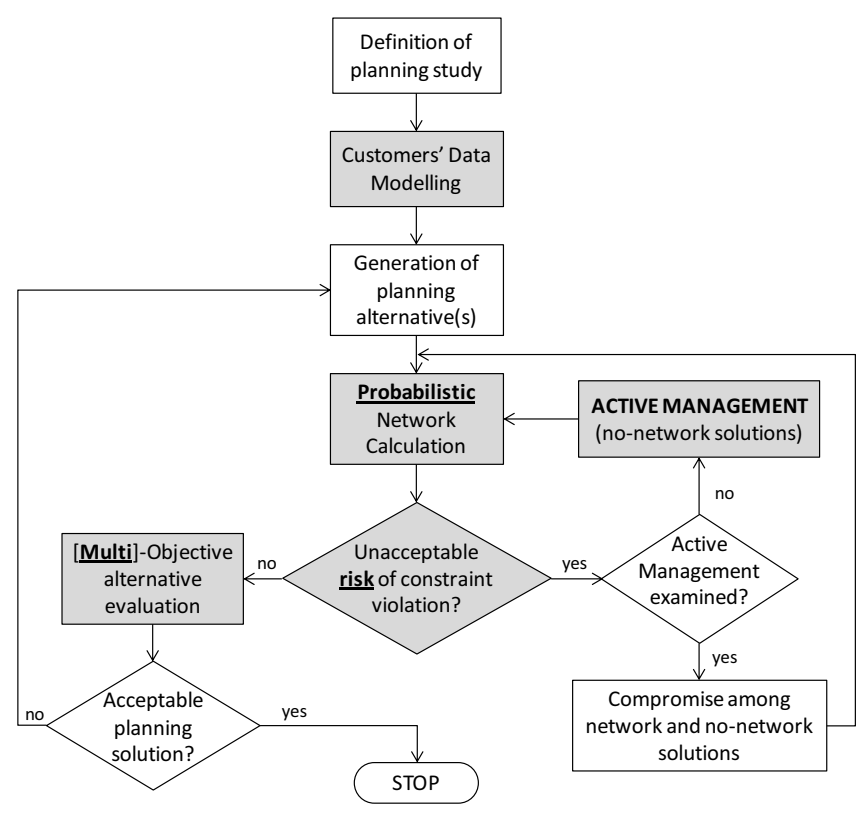

Fig. 2. General procedure for Active Distribution Network planning.

The main novelties are highlighted with grayed blocks and briefly discussed in the following sub-sections.

\section{A. Data modeling}

Traditionally, techniques of load analysis and synthesis were applied to generate characteristic load profiles used to categorize customers into different classes - an approach still used by most distribution utilities. The results of the load analysis were used to produce suitable approximations of the peak demand based on annual energy consumption. This traditional representation was used in distribution planning studies by assuming unique annual values of demand and generation. Peak values were calculated to identifyworst-case operation conditions. They were also used, with a constant annual growth rate, in the fit-andforget approach to plan network expansions for a given horizon. Average values (if necessary) were considered to estimate ohmic losses and for reliability analyses. The main drawback of this deterministic approach is that the distribution network is designed assuming the worst-case conditions as certain, even if actually they have a very low probability of occurrence.

These simplified representations are unsuitable for the planning studies of future ADNs. In order to capture the operational aspects that can affect the planning stage, the variability of demand and generation has to be explicitly represented. The challenge is to find to what extent operational aspects need to be modeled in planning. For instance, while fine granularities (e.g., minute by minute) are capable of capturing detailed operational aspects, when it comes to planning calculations they often prove to be unnecessary and can also lead to time-consuming processes, especially in medium- to long-term horizons. On the other hand, simplistic representations will not bring the benefits from operational strategies to the planning studies, affecting the quality of the results.

Here, the proposed solution is to adopt typical daily patterns to represent the behavior of distribution network customers in a year. These days are then divided into elementary intervals ( 1 hour) and the network calculations are repeated sequentially for each of them. To capture the corresponding uncertainties, loads and DG plants will be modeled with suitable probabilistic density functions.

\section{B. Probabilistic Calculations}

The uncertainties that are inherent to current and future distribution networks suggest both the use of probabilistic models to better represent planning data and the introduction of the risk concept in the selection of planning alternatives. Depending on the adopted stochastic distributions (i.e., Gaussian, Beta, Rayleigh, etc.), network calculations can be performed by tailored probabilistic load flow (PLF) algorithms or the more general Monte Carlo simulation approach. The results of these calculations are the stochastic representations of the nodal voltage and branch current variables. Technical constraints can then be verified with a relative confidence (acceptable risk of violation).

Here, a PLF is used based on the linearization of the load flow equations and of any existing correlation, and the assumption that all random variables are normally distributed. These simplifications are acceptable at medium voltage level and for planning studies given that the demand of substations downstream and the injection from renewable generation are often formed by the aggregation of several elements (customers or generating units). This is also in accordance with the Central Limit Theorem in which loads and generators may be assumed normally distributed.

\section{ADN Implementation}

The first step to integrate ADN solutions into planning is the identification of impacts from control strategies. For instance, operation of automatic voltage controls have small time delays (e.g., 1.5 to 2 minutes), requiring the use of high granularity time-series representation of loads and generators. However, during normal operation, no significant changes are expected on voltages and currents after control actions take place. Therefore, this kind of controls has in general very low impact on planning studies.

Continuous power flow management can have an impact on ohmic losses which constitutes a fundamental performance parameter in distribution networks, affecting Operational Expenditure (OPEX). The potential provision of reactive power support to the transmission system, could also influence the location and sizing of DER, its corresponding revenue (depending on the regulatory environment), and even the network expansion plan.

In general, most ADN solution used to tackle capacity related issues have a direct impact on the Capital Expenditure (CAPEX) as it defers investments that otherwise would be needed. When security of supply requirements, such as $\mathrm{N}-1$ contingencies, are considered, the deferred infrastructure (and therefore CAPEX) could be even more significant.

Considering the above and modeling demand and generation data through daily profiles, it is then possible to technically assess each potential planning solution, generated by the general optimization procedure (Fig. 2).

At first, the network planning solution is pre-sized using existing or minimum allowable cross-sections. Then, for each time interval and network configuration (normal and under contingencies), all nodal currents injected or absorbed are identified from the input data. If operational actions 


\section{Accepted Paper}

from a particular $\mathrm{ADN}$ solution are considered, they have to be integrated at this point to modify the known variables of the PLF (for instance, the optimal scheduling of a distributed energy storage device). The stochastic network calculations should identify the expected values as well as the standard deviations of nodal voltages and branch currents (used to check the risk of constraint violations). If some contingencies appear, the procedure tries to solve them initially through the use of ADN solutions or alternatively with network upgrades. Because the former might require the control of energy resources (generators, storage, loads), its implementation is formalized as a classic Optimal Power Flow (OPF) problem. To reduce the computational burden, this sub-problem can also be simplified (i.e., linearized) and solved with Linear Programming techniques. The outputs of this global procedure that will be used to assess the fitness of the planning solution are:

- expected values of the branch currents, used to evaluate the energy losses;

- network upgrades, that determine CAPEX; and,

- statistics of the ADN interventions, needed to estimate the operational costs of the corresponding solution (e.g., due to the provision of ancillary services).

\section{Multi-Objective Approach}

In the uncertain scenario that characterizes the future ADN, Multi-Objective (MO) approaches could prove to be useful for utilities compared to the traditional single objective function of minimizing the overall network cost. In fact, the impact of several new technologies and control architectures required by $\mathrm{ADNs}$ is hard to characterize exclusively in terms of costs. The lack of clear rules that should govern ADN solutions (e.g., incentives for ancillary services) also adds to its complexity. Therefore, it is easier and more effective to keep separated the various and nonhomogeneous objective functions by comparing known costs with benefits brought about by ADN solutions measured in terms of specific performance indexes. Moreover, the liberalization of the electricity market has broken the vertical monopoly into different players where competition exist at the generation and commercialization ends and regulated monopolies exist at transmission and distribution levels. The need to find good compromises for the conflicting goals of the stakeholders and the difficulty in defining a single objective function is another significant reason that leads to the use of MO approaches.

The goal when solving a multi-objective optimization problem is finding non-dominated solutions (from Pareto optimality concepts) and quantifying the trade-offs in satisfying the different objectives. A solution belongs to the Pareto set if no improvement is possible in one objective without worsening in any other objective. In the literature, several MO methods have been proposed. The complexity of the future distribution system suggests the use of "true" MO algorithms that produce a set of Pareto optimal solutions without the use of subjective weights. These algorithms fall in the group of multi-objective optimization methods based on Evolutionary Algorithms (EA). EA manage sets of possible solutions simultaneously, allowing the immediate identification of several solutions of the Pareto front.
MO programming is a powerful tool but one of its strengths could also be interpreted as a weakness: the provision of more than one solution requires expert interpretation and this might be left open to the subjectivity of the planner. For this reason, this modern approach has to be always combined with the application of Decision Making tools that assign a fitness value to a planning solution representing the overall goodness or risk of its implementation. For the sake of simplicity, a SingleObjective optimization based on the overall cost of the network was used in this work to produce the business cases.

\section{RESUlTS AND DisCUSSION}

The planning tool described in [4] and improved in [6] and [7] has been further developed following the criteria presented in the previous section. This tool has been used to build business cases based on the test network shown in Fig. 3 (which was derived from real data).

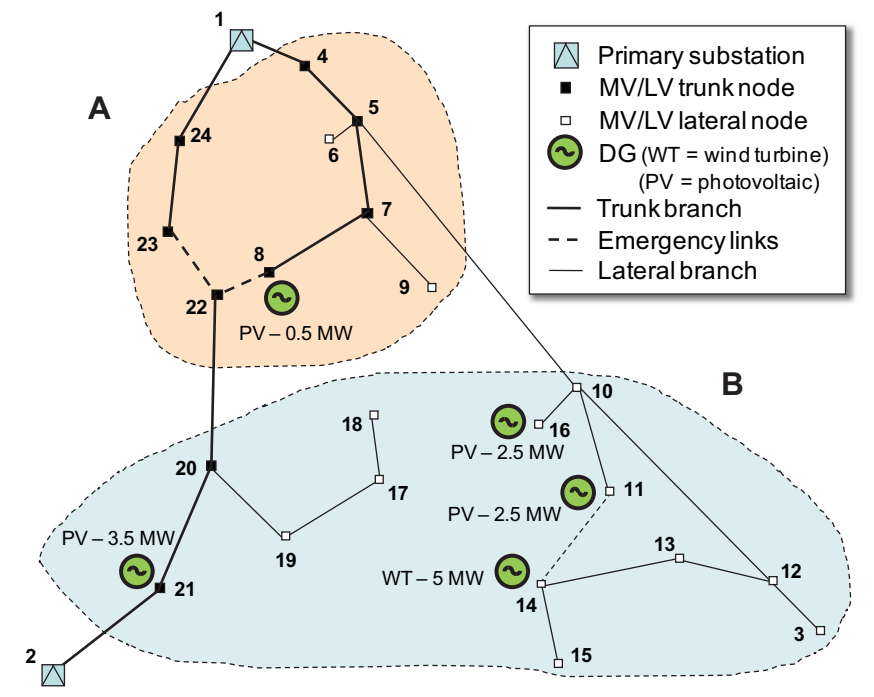

Fig. 3. Case study.

Two primary substations feed $22 \mathrm{MV}$ nodes (9 trunk nodes and 13 lateral nodes) that deliver power to MV and LV customers with a total demand of $13.8 \mathrm{MW}$. The network is operated radially but has open points for reconfiguration purposes. The horizon considered for the planning study is 5 years. In order to stress the network, it is assumed a significant increase in demand (annual growth rate of 3\%). The network comprises two different areas: an urban district (zone A) and a rural area (zone B). In the first one, the total peak urban/industrial load is $9.8 \mathrm{MW}$. Underground cables of $95 \mathrm{~mm}^{2}$ are used due to the high load density and the environmental constraints. Due to the adopted growth rate overloads are expected within the planning horizon. In the rural area, an existing overhead trunk feeder forms the network with $35 \mathrm{~mm}^{2}$ conductors and some lateral branches with $16 \mathrm{~mm}^{2}$ conductors. The long overhead lines and the extended laterals with pure radial structure make these networks electrically weak and, consequently, voltage regulation and overload issues may arise when variable renewable generators are connected. Such a scenario is currently common in many countries because onshore wind farms, in particular, are frequently installed in remote areas, far from urban or industrial 


\section{Accepted Paper}

districts where the load centers are.

The planning studies will consider the connection request of five renewable generators: a $5 \mathrm{MW}$ wind farm and 4 photovoltaic plants. They have been modeled with their typical daily generation profiles that take account of the unpredictability of the primary source by means of normal probabilistic density function. In particular, wind generation is represented with a constant mean output power and a high standard deviation, equal in each hour. The photovoltaic plants have a growing production in the morning, a decreasing production in the afternoon and no production during the night. Their standard deviations are variable hourly (low in the sunrise and sunset, and high in the midday hours).

Five different planning approaches have been considered in the paper and compared in terms of network CAPEX and quality of the solution:

1.F\&F. Traditional fit and forget;

2.Prob_5\%. F\&F combined with a probabilistic approach with a low (5\%) accepted risk of constraint violation;

3.Prob_20\%. F\&F combined with a probabilistic approach with a high (20\%) accepted risk of constraint violation;

4.ADN_PQ. ADN with control of DG active and reactive power and a $20 \%$ accepted risk of constraint violation;

5.DES. ADN with installation of distributed electricity storage (DES, $3 \mathrm{MW}-8 \mathrm{~h}$ ) in the lateral node 10 and a $20 \%$ accepted risk of constraint violation.

Fig. 4 shows the CAPEX for each of the above approaches. As expected, the F\&F approach is the most expensive one due to the need of complying with the technical constraints for the most, but extremely rare, severe conditions both in normal and post-contingency network configurations. The overloaded assets in the urban district resulted in the duplication of the trunk feeder from the primary substation 1 to the node 7 (operating the branch between secondary substations 5 and 7 as an emergency link) as well as the reinforcement of existing underground cables (now with a $150 \mathrm{~mm}^{2}$ cross section). This strong intervention on the urban network allows limiting the excessive voltage rise on the long rural lateral fed by secondary substation 5 . On the other hand, the rural network supplied by the primary substation 2 has voltage issues and needs to reinforce the overhead trunk feeder from $35 \mathrm{~mm}^{2}$ to $70 \mathrm{~mm}^{2}$.

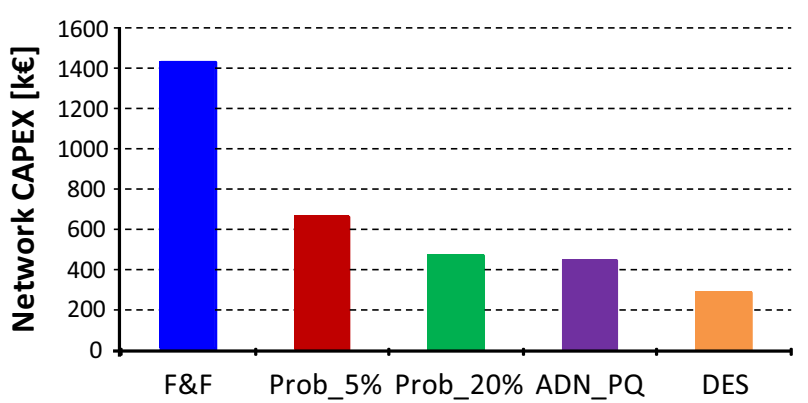

Fig. 4. Network CAPEX comparison among different planning approaches.

A more effective approach to plan the distribution network in a strong uncertain scenario due to the presence of renewable generation is to take account of the occurrence probability of each operating condition by using probabilistic calculations and identifying an acceptable risk of constraint violation. With a $5 \%$ of acceptable risk (Prob 5\%) the network CAPEX is more than halved, while with a higher risk (i.e., Prob_20\%) it is reduced almost to one third of the F\&F cost (Fig. 4). In the first hypothesis, the low level of risk accepted requires reinforcing the urban trunk feeder (from $95 \mathrm{~mm}^{2}$ to $240 \mathrm{~mm}^{2}$ ) and several lateral branches (from $16 \mathrm{~mm}^{2}$ to $35 \mathrm{~mm}^{2}$ ). The lower investment is a consequence of not requiring a new line. With the acceptance of a higher risk, fewer upgrades are needed (in particular the link between nodes 5 and 10 is not upgraded and other laterals are only refurbished to $25 \mathrm{~mm}^{2}$ ). It is worth noticing that, even if the solutions obtained are less expensive, the planning results of the probabilistic approach are still of good quality as shown below. Fig. 5 compares the voltage profile of the right hand-side feeder (in Fig. 3) during normal operation (i.e., no branch out of service).

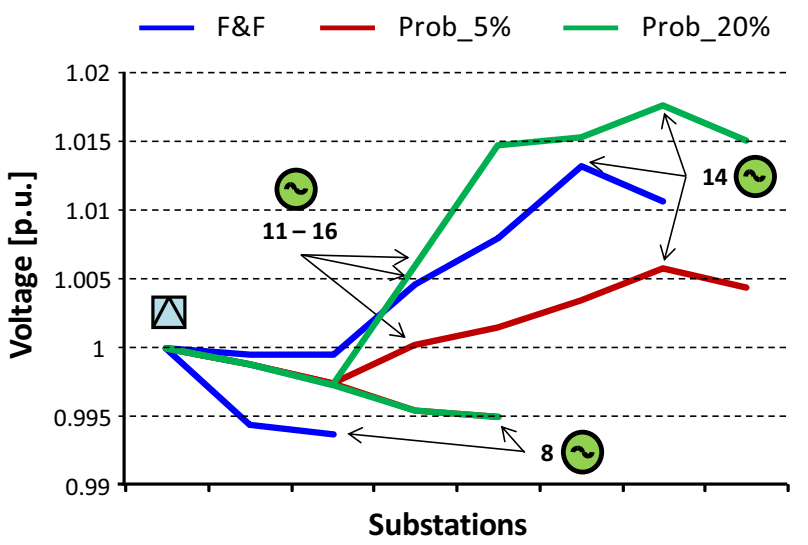

Fig. 5. The voltage profile in planning alternatives with passive operation.

Despite some differences, the operating conditions are quite similar with a maximum voltage rise (average values) that remains lower the $2 \%$. Also analyzing the extreme conditions that appear when the network is reconfigured due to the disconnection of a faulted branch (Fig. 6), the voltages stay practically always within the prefixed limits (assumed to be $\pm 8 \%$ of the maximum voltage variation with respect to the nominal value, and $16 \%$ with postcontingency configurations). The values represented in the figures refer to the mean value of the nodal voltage calculated during the worst configuration and the more stressful hour for each node. Considering the corresponding standard deviation, some instances can exist when the limits are exceeded. However, these are extremely rare and last only for a few hours in which repairs take place. Consequently, these voltage issues may be acceptable for the DNO. 

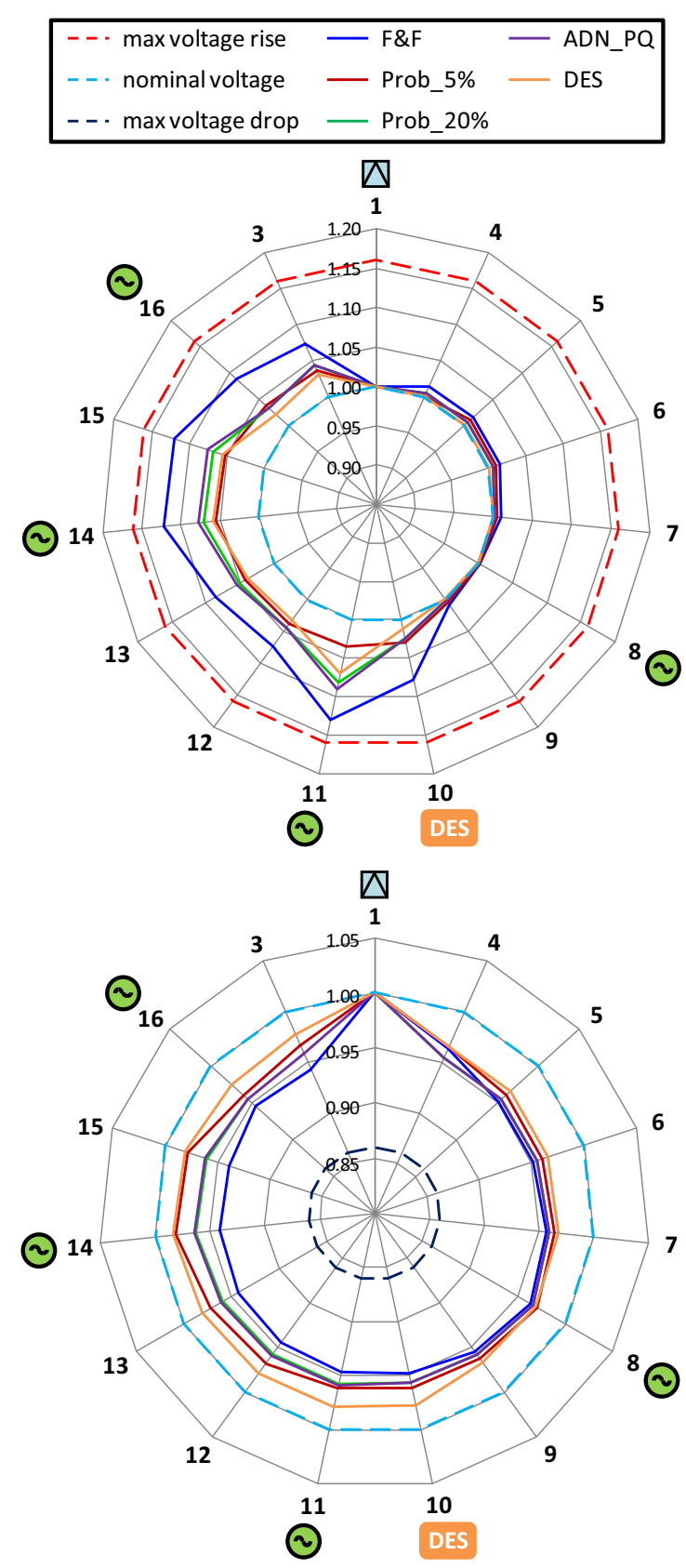

Fig. 6. Maximum and minimum nodal voltages during the worst operating conditions.

The last two planning approaches (i.e., ADN_PQ and DES) have been obtained considering different implementations of active management. ADN_PQ uses an active control for the power output of all the generators. By exploiting the technology available in many DG plants, it is assumed that the DNO can request DG operators to inject/absorb reactive power and/or to limit the active power whenever severe operating conditions appear in the network. For the test case used in this paper, this solution brings an additional but minor reduction of the CAPEX if compared to Prob_20\% (passive management and the same acceptable risk). In fact, the active management allows just avoiding few lateral upgrades with a very low probability of generation curtailment for DG located at nodes 11 and 14 . These rare interventions of the DNO are determined by the occurrence of overloads on branch 5-10. The nature of the constraint violation (overload and not overvoltage) justifies the control only of the active power generated and not of the reactive power. The absence of controllable energy resources in the urban portion of the network is the reason why the active management cannot avoid the reinforcement of the trunk feeder. The voltage profile patterns are similar to those shown in Fig. 6.

The last planning alternative, DES, exploits the installation of an electricity storage device connected to the secondary substation 10 . The network investment is further reduced in comparison with the other planning cases (Fig. 4). In general, DES reduces losses by avoiding excessive reverse power flows caused by renewable generation. By artificially increasing the neighboring load with DES, the excess power is used close to the DG plants and not reversed to the HV system, resulting in a significant improvement of the efficiency. Furthermore, network upgrades are reduced because DES increases the homotheticity (or positive correlation) between the power generated and the load, making reinforcements less necessary (the storage's charge pattern follow the PV production).In conclusion, the use of DES is useful to increase the DG hosting capacity in distribution systems even without active controls of generators in place. The DNO has only to control DES resources and operate the network without any sharing of responsibilities with producers. The voltage profiles were the best achieved both in the normal and post-contingency configurations (see Fig. 6).

\section{CONCLUSIONS}

From the analysis of the presented results it can be stated that with the right tools more effective distribution network planning solutions both in terms of investments and network performances can be achieved. Moreover, the capability to correctly assess the value of the active operation in the distribution planning is fundamental for the convincement of the DNOs to move towards the future Smart Grid concept.

\section{REFERENCES}

[1] CIGRE WG SC6.11, "Development and operation of active distribution networks", CIGRE Technical Brochure, April 2011.

[2] G. Celli, E. Ghiani, F. Pilo and G.G. Soma, "New electricity distribution network planning approaches for integrating renewable", WIREs Energy Environ, to be published.

[3] K. E. Bakari, C. Carter-Brown, S. Jupe, A. Baitch, C. Abbey, A. R. Aoki, F. Mingtian, C. Nakazawa, and F. Pilo, "Survey on methods and tools for planning of active distribution networks", in Proc. 2012 CIRED Workshop.

[4] G. Celli, F. Pilo, G. Pisano, and G.G. Soma, "Optimal planning of active networks", in Proc. $200816^{\text {th }}$ Power Systems Computation Conference.

[5] R. A. Walling, R. Saint, R. C. Dugan, J. Burke, and L. A. Kojovic, "Summary of distributed resources impact on power delivery systems," IEEE Trans. on Power Systems, vol. 23, no. 3, pp. 16361644, July 2008.

[6] G. Celli, S. Mocci, F. Pilo, M. Loddo, "Optimal Integration of Energy Storage in Distribution Networks", in Proc. 2009 PowerTech Conference.

[7] G. Celli, S. Mocci, F. Pilo, G.G. Soma, "Multi-Objective programming for optimal DG integration in active Distribution Systems", in Proc. 2010 IEEE-PES General Meeting. 